\title{
NON-BANDLIMITED RESAMPLING OF IMAGES
}

\author{
Beilei Huang \\ Nanyang Technological University \\ School of Computer Engineering \\ Singapore 639798
}

\begin{abstract}
The resampling of discrete-time signals where the underlying ana$\log$ signal is non-bandlimited is considered in this paper. We extend the generalized sampling theory developed based on the principle of consistency to resampling. Realizing the resampling system has both discrete input and output, the performance of the resampling filter is considered in $l^{2}$ instead of the traditionally used $L^{2}$. We show that the performance of the resampling system depends on the resampling rate instead of the actual interpolating kernels. The theory can be applied to image processing applications like zooming to provide better response to high frequency components. Since the resampling process is discrete in nature, our filter designed to optimize resampling in $l^{2}$ is shown to outperform other techniques designed in $L^{2}$.
\end{abstract}

\section{INTRODUCTION}

Most of the existing techniques for processing discrete-time signals are based on the (implicit) assumption that the underlying analog signals are bandlimited. This assumption does not hold for most images where sharp edges exists. Therefore, even simple operations like the enlargement of an image is not trivial since aliasing will be introduced through the interpolation filter.

A recent method proposed in [1] tries to overcome this problem partially by representing the interpolation and resampling kernels as polyphase filters. The frequency responses of the polyphase filter are equalized and therefore aliasing is reduced. However, high frequency information will still be lost in the process and is unrecoverable. Other techniques have been introduced to prevent distortion as well as reserve the high frequency components [2, 3]. The underlying principle used in these methods is the Generalized Sampling Theory (GST) for non-bandlimited continuous-time signals [4]. However, as pointed out in [5], the filters designed based on the GST is optimized for an operator defined in $l^{2} \rightarrow L^{2}$; it can not be directly applied to the resampling system, which is associated with an operator for $l^{2} \rightarrow l^{2}$. Furthermore,the filter designed based on GST that minimizes the $L^{2}$ error does not necessarily minimize the $l^{2}$ error. Since the generalized sampling theory is developed to optimize reconstruction, it can be suboptimal when applied to resampling.

In this paper, we consider the resampling of discrete-time signals where the underlying analog signal is non-bandlimited. Our filter is designed to optimize the resampling process in the discrete domain. The main contributions of this paper are as follows. First, we proved that the performance of the resampling system depends on the resampling rate instead of the interpolating kernel. Second, we extended the original GST to resampling and the correction filters obtained are

\author{
Edmund M-K. Lai \\ Massey University \\ Institute of Information Science and Technology \\ Wellington, New Zealand
}

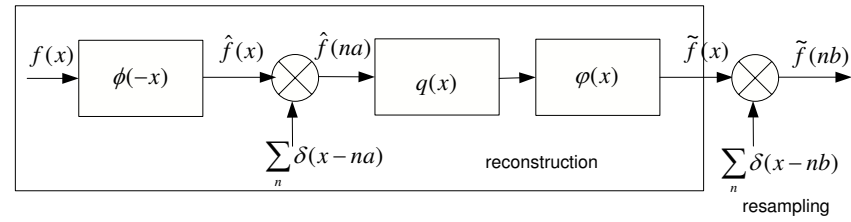

Fig. 1. Block diagram representation of reconstruction and resampling system.

optimal for the resampling process. We show by through an example of image enlargement that the our the filter based on our extended GST performs better than the one defined by the original GST.

This paper is organized as follows. The generalized sampling theory is reviewed in Section 2. In Section 3, this theory is extended to resampling systems and the correction filter is redefined subject to the new optimizing criteria defined in discrete domain. The image enlargement example is discussed in Section 4.

\section{REVIEW OF GENERALIZED SAMPLING THEORY}

The schematic diagram of the generalized sampling theory is shown in Figure 1. Since most of real time signals are energy limited, we restrict our discussion to the Hilbert space $L^{2}$.

Define the translation operator $T_{a}: L^{2} \rightarrow L^{2}, T_{a} \phi(x)=\phi(x-$ $a)$; the set of uniformly shifted vectors $\left\{T_{n a} \phi(x)\right\}_{n \in Z}$ consists of a frame for its closed linear space

$$
V(\phi)=\operatorname{span}\left(\left\{T_{n a} \phi(x)\right\}_{n \in Z}\right)
$$

In generalized sampling theory, the uniform samples $\widehat{f}(n)$ of an analog signal $f(x)$ are viewed as the inner product of $f(x)$ with a set of vectors $\left\{T_{n a} \phi(x)\right\}_{n \in Z}$

$$
\widehat{f}(n)=f(x) * \phi(-x) \cdot \delta(x-n a)=\left\langle f(x), T_{n a} \phi(x)\right\rangle
$$

Similarly, the reconstruction of $\widetilde{f}(x)$ using $\widehat{f}(n)$ is performed using vectors spanning the reconstruction subspace $V(\varphi)$

$$
\widetilde{f}(x)=\sum_{n} \widehat{f}(n) T_{n a} \varphi(x)=\sum_{n}\left\langle f(x), T_{n a} \phi(x)\right\rangle T_{n a} \varphi(x)
$$

Therefore perfect reconstruction can only be achieved when $f(x) \in$ $V(\varphi)$ and $\left\langle T_{m a} \phi(x), T_{n a} \varphi(x)\right\rangle=\delta_{m, n}$. If this condition is satisfied, the acquisition filter $\phi(x)$ is called the ideal prefilter of $\varphi(x)$. Otherwise, reconstruction errors (aliasing, distortion) would be inevitable. 
The GST loose the constraint from perfection reconstruction to consistent reconstruction. It requires that if $\widetilde{f}(x)$ is re-injected into the system, the reconstructed signal should produce the same measurements $\widehat{f}(n a)$ as the original input. This can be achieved by using a correction filter $Q$ to modified the coefficient set such that the signal is projected onto $V(\varphi)$ orthogonal to $V(\phi)$ [4]. Therefore, the reconstruction error $\|\widetilde{f}(x)-f(x)\|_{L^{2}}$ will be minimized for the system with arbitrary $\phi(x)$ and $\varphi(x)$.

Let the cross correlation between $\phi(x)$ and $\varphi(x)$ is defined by

$$
A_{\phi \varphi}(k)=\langle\phi(x-k), \varphi(x)\rangle
$$

If it is invertible, then the correction filter $Q$ is specified via the inversion of $A_{\phi \varphi}$ in the $z$ transform domain:

$$
Q(z)=A_{\phi \varphi}^{-1}(z)
$$

The generalized sampling theory can be described as

$$
\forall f(x) \in L^{2}, \tilde{f}(x)=\sum_{n}\left\langle f(x), T_{n a} \phi\right\rangle\left(A_{\phi \varphi}^{-1} * T_{n a} \varphi\right)(x)
$$

The term $\left(A_{\phi \varphi}^{-1} * T_{n a} \varphi\right)(x)$ can be interpreted as the quasi-dual operator of the acquisition filter, denoted by $\widetilde{\phi}$. It can be verified that $\widetilde{\phi}(x)$ and $\phi(x)$ satisfies

$$
\left\langle T_{n a} \phi, T_{m a} \widetilde{\phi}\right\rangle=\delta_{m, n}
$$

There are two advantages of using GST in resampling system. First, by using non bandlimited kernels, the high frequency components can be preserved. Second, it ensures minimum mean squared error when non-ideal operators are used. The resampling system can be viewed as a two-step process: an implicit reconstruction process followed by a sampling process [6]. However, as we shall see in Section 3 , the filter designed based on GST that minimizes the $L^{2}$ error does not necessarily minimize the $l^{2}$ error. Since the generalized sampling theory is developed to optimize reconstruction, it can be suboptimal when applied to resampling.

\section{THE RESAMPLING SYSTEM}

The resampling system differs from the reconstruction system by an additional sampling stage as shown in Figure 1. Its discrete output $\widetilde{f}(m)$ is obtained by sampling $\widetilde{f}(x)$ at a rate $b$ which is typically different from the original sampling rate $a$. The output of the resampling system is given by

$$
\widetilde{f}_{b}(m)=\sum_{n}(q * \widehat{f})(n a) T_{n a} \varphi(m b)
$$

The subscript $b$ is used to indicate the sampling rate of the sequence and is often omitted if it is clear. Obviously, the output $\widetilde{f}(m) \in$ $V\left(\varphi_{b}\right)$ where

$$
V\left(\varphi_{b}\right)=\operatorname{Span}\left\{T_{n a} \varphi(m b)\right\}_{m, n \in Z}
$$

Before we state the conclusion, we start with a fundamental result:

Proposition 1. Sampling any continuous function $h(x) \in L^{2}$ at rate $b$, the discrete sequence $h(m)=h(x)_{x=m b}, m \in Z$ generates the same space as the pulse train $\Delta_{b}=\sum_{n} \delta(x-n b)$ iff $h(m) \neq 0$ for any $m$.
Actually this statement requires $\sum_{m} h(m)$ to define an invertible convolution operator. Since

$$
\sum_{m} h(m)=\sum_{m} \int h(x) \delta(x-m b) d x
$$

hence $\|h(m)\|_{l^{2}} \leq\|h(x)\|_{L^{2}}<\infty$. The sequence $h(m)$ defines a bounded sequence and thus is invertible provided any of the $h(m)$ is nonzero.

This proposition gives us some distinctive properties of the resampling system. The resampling system deals with output in discrete space generated by shifts of the sequence $\varphi_{b}(m)$, while reconstruction system deals with continuous output in the space generated by $\left\{T_{n a} \varphi(x)\right\}_{n \in Z}$. According to the Proposition 1, any sequence sampled at $b$ falls in the discrete space generated by the pulse train $\Delta_{b}$ despite of the specific choices of $\varphi(x)$. That is for all sequence $h 1_{b}(m)$, it can be represented by a weighted sum of another sequence $h 2_{b}(m)$ despite $h 1(x)$ and $h 2(x)$. Therefore, we obtain an outstanding conclusion in the next proposition

Proposition 2. Given two sampling rate $a$ and $b$ and the predefined prefilter $\phi(-x)$, the resampling system can always achieves the same performance with properly designed correction filter for different choices of $\varphi(x)$.

The performance of any resampling system depends on the difference between acquisition space and the resampling space. For given $a$ and $\phi(-x)$, according to (1), the acquisition space is defined. On the other hand, since all $\varphi_{b}(m)$ belongs to the same space, $\left\{T_{n a} h_{b}(m)\right\}$ also spans the same resampling space. Therefore Proposition 2 is justified.

For the special case $a=b$,

$$
\operatorname{span}\left(\left\{T_{n a} \varphi_{b}(m)\right\}_{n, m \in Z}\right)=\operatorname{span}\left(\left\{\varphi_{b}(m)\right\}_{m \in Z}\right)
$$

No matter how $\phi(x), \varphi(x) \in L^{2}(\mathbb{R})$ is chosen, the system should achieve perfect performance in the sense that the discrete input $\widehat{f}_{a}(n)$ can be perfectly reconstructed. When $a \neq b$, the acquisition space and the synthesis space are generally different. Similar to the reconstruction system, a correction filter defined the operation $l^{2} \rightarrow l^{2}$ can be used to project the signal representation $\widehat{f}(n a) V\left(\phi_{a}\right)$ onto $V\left(\varphi_{b}\right)$. Our next task is to define the correction filter used to achieve minimum error in $l^{2}$. There are two types of resampling system to be treated separately, one is of continuous input, or an system defined from $L^{2} \rightarrow l^{2}$; the other one is of discrete input defining a system of $l^{2} \rightarrow l^{2}$.

\subsection{When $\phi(x) \neq \delta(x)$}

Similar to the correction filter defined in the reconstruction case, there is one unique correction filter in resampling system such that the principle of consistency is satisfied.

Theorem 1. Consider the resampling system shown in Figure 1 with $\phi(-x) \neq \delta(x)$. Define the discrete cross correlation function as

$$
A_{\phi \varphi}(k a)=\sum_{m} \varphi_{b}(m) \phi(m b-k a)
$$

If $A_{\phi \varphi}(\mathrm{ka})$ is an invertible operator from $l_{2} \rightarrow l_{2}$, there exist a correction filter $q=A_{\phi \varphi}^{-1}$ in $z$ transform domain such that the principle of consistency is satisfied:

$$
\left\langle g(x), T_{n a} \phi(x)\right\rangle=\left\langle\sum_{m} \widetilde{f}_{b}(m), T_{n a} \phi(x)\right\rangle
$$


Proof. By the principle of consistency, when $\tilde{f}(m b)$ is re-injected to the system, it should produce the same set of samples $\widehat{f}(n a)$. Thus, it requires that

$$
\begin{aligned}
\widehat{f}(n a) & = \\
& =\sum_{n}(q * \widehat{f})(n a) \sum_{m} \varphi(m b-n a) \phi(m b-k a)
\end{aligned}
$$

By substitute (7) into the equation, the consistency requirement (11) is reduced to

$$
\widehat{f}(n)=q * \widehat{f} * A_{\phi \varphi}(n)
$$

Therefore, the discrete filter $q$ is defined to be $A_{\phi \varphi}^{-1}$.

\subsection{When $\phi(x)=\delta(x)$}

The reconstruction system is well defined when the input is either discrete or continuous. However, in case of the resampling system, the principle of consistency is not directly applicable under certain circumstances. Suppose that $\phi(x)=\delta(x)$; the consistency principle requires that when $\sum_{m} \widetilde{f}_{b}(m)$ is resent to the system, its samples at $\sum_{n} \delta(x-n a)$ should be the same as the original continuous signal does. This is to sample a discrete sequence $\widetilde{f}_{b}(m)$ at a different rate $a$, which is generally not accomplishable.

Therefore, the principle of consistency has to be modified to be adaptable in the resampling system. Referring to Figure 1, $f(x)=$ $\widehat{f}(x)$ since $\phi(x)=\delta(x)$. Denote the samples $\widehat{f}(n)=f_{a}(n)$ and the shifted filter $\varphi_{b}(x-m)=\varphi(x-m b)$. The consistency principle is restated as

Principle of consistency: The sequence $\widetilde{f}_{b}(m)$, if re-injected to the system, should appear the same as the sequence $\left.\widehat{f}_{(} n\right)$ to produce the same output, or

$$
\sum_{m} \tilde{f}_{b}(m) * q * \varphi(x)=\widetilde{f}(x)
$$

In reconstruction system, the principle of consistency requires that the output should appear the same as the input to the same acquisition space. Therefore, if $\widetilde{f}$ is resent to the system, the output should nonetheless be $\widetilde{f}$. The same idea is applied in the resampling system to derive the condition in (13), which is one step further from "the same measurement of the acquisition filter" to "the same output of the reconstruction filter".

From the modified consistency requirement, the correction filter can be obtained through the following theorem.

Theorem 2. For resampling system with $\phi(x)=\delta(x)$, the principle of consistency can be satisfied uniquely iff $\varphi(k b)$ is invertible. The correction filter then can be defined as $Q(z)=\Psi_{b}^{-1}(z)$.

Proof. Substitute (7) into (13) and we have

$$
\sum_{m}\left(\tilde{f}_{b} * q\right) \varphi_{b}(x-m)=\sum_{n}\left(f_{a} * q\right)(n) \varphi_{a}(x-n)
$$

substitute (7) into the LHS of the above equation and it can be rewritten as

$$
\begin{aligned}
& \sum_{m}\left(\tilde{f}_{b} * q\right) \varphi_{b}(x-m) \\
= & \sum_{n}\left(f_{a} * q\right)(n) \sum_{m} \sum_{k} \varphi_{b}(x-m) \varphi_{a}(k b-n) q(m-(\sharp \bar{J})
\end{aligned}
$$

Compare (15) with the RHS of (14), the consistency is satisfied with

$$
\sum_{k} \sum_{m} \varphi(k b) q(m-k) \varphi(x-m b)=\varphi(x)
$$

or

$$
\sum_{k} \varphi_{b}(k) q(m-k)=\delta_{m, 0}
$$

Therefore, the correction filter is defined as the inverse of the reconstruction filter, sampling at output rate.

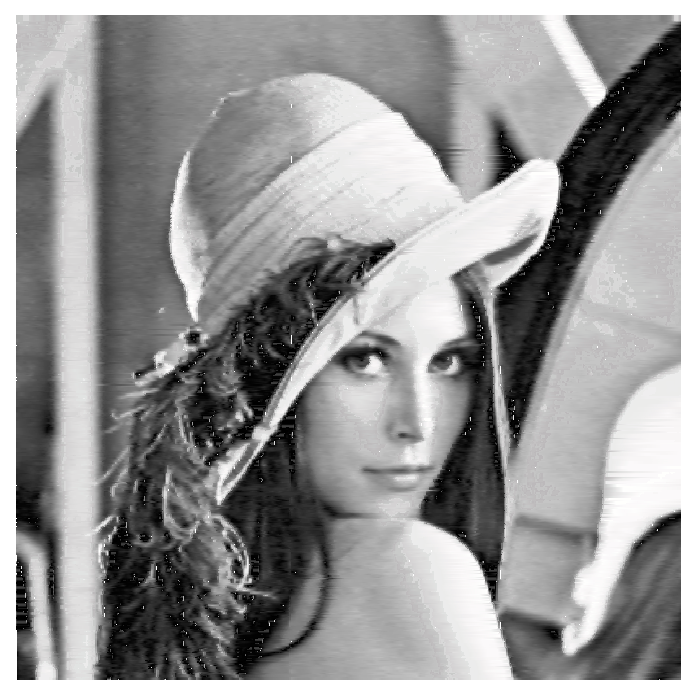

Fig. 2. Enlargement by 2. The bilinear interpolator and correction filter optimized error in $L^{2}$ is used

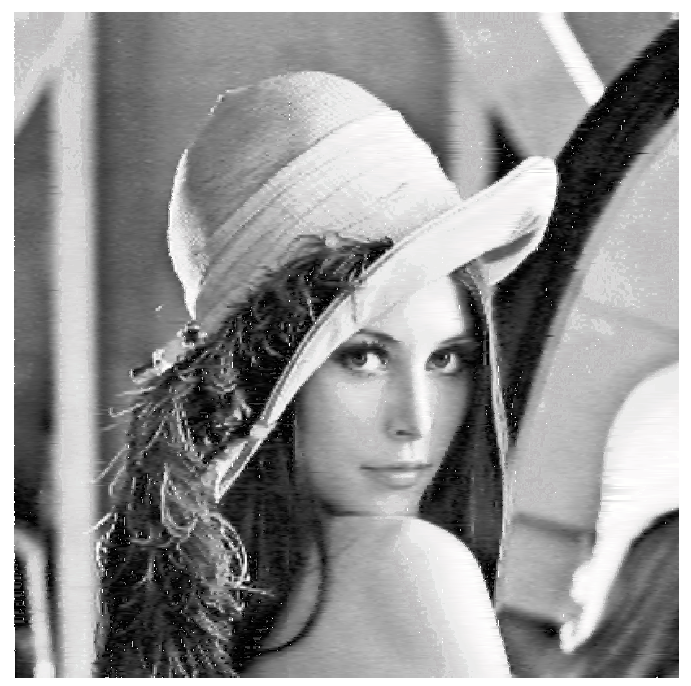

Fig. 3. Enlargement by 2. The bilinear interpolator and correction filter optimized error in $l^{2}$ is used

To generalize the two cases above, we find that when $\phi(x)=$ $\delta(x)$, the correction filter can be calculated using (10) with $\phi(x)=$ 1. Comparing (4) and (10), the correction filter used in the resampling case depends much on the resampling rate $b$ chosen. Because 
of this, the correction filter for resampling can be very different from the one obtained based on GST, even if the acquisition and synthesis filters are the same.

In summary, the design of correction filter in the resampling system is subject to the requirement that the discrete output should be consistent with the input, when re-injected into the system. The correction filter projects the input signal, either continuous or discrete onto the space of $V\left(\varphi_{b}\right)$ such that the different between input and output measured in $l^{2}$ is minimized. This criteria is more suitable for resampling system where both input and output can be discrete.

\section{EXAMPLE}

The theory developed above is applied to the example of image zooming. The interpolator used in image processing applications are generally small kernels like linear or cubic interpolators. Because they are short in time, their frequency response is bad and will cause aliasing and distortion which would degrade the quality of the image. The correction filter defined in Theorem 2 is inserted and the performance of zooming of factor 2 with and without the correction filter is shown in Figure 2 and Figure 3.

We clearly see the improvement of high frequency response due to the correction filter, which includes the edges of the hat edge, the feather decorations and hair. The overall distortion of the image is also reduced as can be observed from the improvement in the texture of the hat and the face.

\section{CONCLUSION}

In this paper, we developed an extension to the generalized sampling theory so that it can be applied to resampling of discrete-time signals. The underlying analog signal can be non-bandlimited, therefore our theory could provide better response to the high frequency signal. We prove that the performance of the resampling system depends on the resampling rate instead of the interpolating kernels used. A correction filter is designed to optimize the $l^{2}$ error instead of the commonly used $L^{2}$ error. It shows that our filter has better response when applied to applications like image zooming than other filters used to optimize $L^{2}$ error.

\section{REFERENCES}

[1] D. Seidner, "Polyphase antialiasing in resampling of images," IEEE Trans. Image Processing, vol. 14, no. 11, pp. 1876-1889, Nov. 2005.

[2] M. Unser, A. Aldroubi, and M. Eden, "Enlargement or reduction of digital images with minimum loss of information," IEEE Trans. Image Processing, vol. 4, no. 3, pp. 247-258, Mar. 1995.

[3] C. Lee, M. Eden, and M. Unser, "High-quality image resizing using oblique projection opreators," IEEE Trans. Image Processing, vol. 7, no. 5, pp. 679-692, May 1998.

[4] M. Unser and A. Aldroubi, "A general sampling theory for nonideal acquisition devices," IEEE Trans. Signal Processing, vol. 42, no. 11, pp. 2915-2925, November 1994.

[5] A.J.E.M. Janssen and T. Kalker, "A note on Unser-Zerubia generalized sampling theory applied to the linear interpolator," IEEE Trans. Signal Processing, vol. 47, no. 8, pp. 2332-2335, August 1999.

[6] T.M. Lehmann, C. Gonner, and K. Spitzer, "Survey: Interpolation methods in medical image processing," IEEE Trans. Med. Imag., vol. 18, no. 11, pp. 1049-1075, Nov. 1999. 